\title{
EP-161
}

\section{Functional neuroendocrine tumours of pancreas}

\author{
Prabhat NICHKAODE*, Sai Charan Reddy MALLELA, Balaji Prathep SANTHAN, Manichandra Reddy CHAMAKURA, Hara Pradeep RAYANI
}

Department of General Surgery, Dr. D.Y. Patil Vidyapeeth, Pune, India

Introduction: Fascinating small \& Rare Tumors of Pancreas. Incidence less than 1 per 1 lakh person per year. Puzzling clinical presentation. Excellent outcome when picked early \& treated. Surgery mainstay of resectable tumors. Diagnostic modalities range from simple to highly technical methods but Diagnosis, localization \& Treatment clearly defined. No longer a matter of debate.

Methods: We studied the Clinical Spectrum of Functional NET of Pancreas in medical college Hospital. As far Localization \& diagnostic modalities. To analyze management \& outcome. Feasibility To Undertake Such Surgeries In Hospital Without a Specialty Department. Retrospective analysis of 27 Histopathologically proven Functional NET of Pancreas - from 1998 to 2020. Demographic data, Clinical presentation, Tumor characteristics, Management \& Outcome were analyzed.

Results: The most common NET was Insulinoma, followed by other tumors like gastrinoma, and Glucaganoma. USG-Sensitivity small tumors: $10 \%$ to $30 \%$, Liver Mets: $90 \%$. CT with/without angiography. MRI. Intraoperative USG- sensitivity very high, Palpatory Method- very useful. PET with C11-\%HTP or C11 L-Dopa, are recent investigations. General tumor markers Chromogranin A- 90 to $100 \%$, Synaptophycin, Pancreatic Polypeptide- $40 \%$ to $60 \%$ HCG /Alpha/Beta-15\% to $20 \%$.

Conclusions: Treatment is either enucleation or pancreatic resection depending on size of tumor. Enucleation has the Benefit of sparing normal pancreatic parenchyma as long as integrity of $\mathrm{PD}$ is preserved. Laparoscopic resection of pancreatic endocrine tumors is becoming common, for patients with unresectable disease, new somatostatin analog -LANREOTIDE- remains biologically active for up to two weeks following a single injection and controls symptoms. 